\title{
A new type of oculocutaneous albinism with a novel OCA2 mutation
}

\author{
Sang Yoon Lee ${ }^{1}$, Eun Joo Lee ${ }^{1}$, Jun Chul Byun ${ }^{2}$, Kyung Mi Jang ${ }^{3}$, Sae Yoon Kim ${ }^{3}$, Su-Kyeong Hwang ${ }^{4}$ \\ ${ }^{1}$ Department of Pediatrics, Kyungpook National University Hospital, Daegu, Korea \\ ${ }^{2}$ Department of Pediatrics, Daegu Fatima Hospital, Daegu, Korea \\ ${ }^{3}$ Departments of Pediatrics, Yeungnam University College of Medicine, Daegu, Korea \\ ${ }^{4}$ Department of Pediatrics, School of Medicine, Kyungpook National University, Daegu, Korea
}

Received: May 12, 2020

Revised: June 14, 2020

Accepted: June 15, 2020

Corresponding author:

Su-Kyeong Hwang, MD, PhD

Department of Pediatrics, School of

Medicine, Kyungpook National

University, 807 Hoguk-ro, Buk-gu,

Daegu 41404, Korea

Tel: $+82-53-200-5704$

Fax: +82-53-425-6683

E-mail: skhwang@knu.ac.kr
Oculocutaneous albinism (OCA) is a group of rare genetically heterogeneous disorders, characterized by hypopigmentation of the eyes, skin, and hair, which result in ocular abnormalities and a risk of developing skin cancer. Currently, there is no ophthalmologic procedure or drug that prevents the clinical features of OCA. Here, we report a new type of OCA in two, unrelated Korean families with the same OCA2 mutation. Affected individuals in this study are different from those of previous reports in two aspects: an inheritance pattern and clinical presentation. All reported patients with OCA have shown an autosomal recessive inheritance pattern, while our patients showed an autosomal dominant inheritance pattern. Small amounts of pigment can be acquired with age in OCA, but there is no substantial variation from adolescence to adulthood in this regard. A case where the patient attained normal pigmentation levels has never been reported. However, our patients displayed completely normal pigmentation in their late twenties. Whole exome sequencing and in-silico analysis revealed a novel mutation, OCA2 c.2338G $>$ A p.(G780S) (NM_000275) with a high likelihood of pathogenicity. Sanger sequencing of p.G780S identified the same mutation in the affected individuals, which was not found in the family members with normal phenotype. We hypothesize that OCA2 G780S not only acts as a pathogenic variant of OCA but also induces pigmentation by enhancing the melanogenesis gene expression of other modifier genes, such as SLC45A2 and TPC2. These findings may provide further understanding of melanin biosynthesis and new treatment methods for OCA.

Keywords: Mutation; Oculocutaneous albinism; Whole exome sequencing

\section{Introduction}

Oculocutaneous albinism (OCA) is a group of rare genetically heterogeneous disorders. OCA is an autosomal recessive disorder associated with mutations in genes which control the biosynthesis of the melanin pigment [1]. The pigmentary system is dependent on the production of melanin, the light-absorbing biopolymer found within ocular, epidermal, and follicular melanocytes [2]. OCA is characterized by hypopigmentation of the eyes, skin and hair, which results in ocular abnormalities and a risk of developing skin cancer. All patients with OCA are accompanied by ocular abnormalities, such as nystagmus, reduced visual acuity, photophobia, strabismus, foveal hypoplasia, hypopigmentation of the iris, and color vision impairment [3]. Currently, there is no ophthalmologic

Copyright (C) 2021 Yeungnam University College of Medicine

This is an Open Access article distributed under the terms of the Creative Commons Attribution Non-Commercial License (http://creativecommons.org/licenses/by-nc/4.0/) which permits unrestricted non-commercial use, distribution, and reproduction in any medium, provided the original work is properly cited. 
procedure or drug that prevents the clinical features of OCA. The clinical spectrum of OCA varies, ranging from mild hypomelanosis to complete lack of melanin production, which is mainly associated with tyrosine metabolism [4].

To-date, seven types of OCA (OCA1-7) have been identified, depending on a different genetic defect. $O C A 2$, which is the most common type of albinism worldwide [5], is caused by pathogenic variants of OCA2 gene [6]. The OCA2 locus maps to chromosome $15 \mathrm{q}$ and is the human homolog of the mouse pink-eyed dilution gene encoding the $P$ protein [7-9]. The mutation types causing OCA 2 are variable, including missense/nonsense single nucleotide variations (SNVs), splicing variations, small indels, and large indels. So far, 184 relevant mutations have been reported in $\mathrm{Hu}$ man Gene Mutation Database (HGMD, professional 2019.4). The most common OCA2 mutation is a $2.7-\mathrm{kb}$ deletion on exon 7 , which is found in many affected individuals of sub-Saharan African descent [10]. Other OCA2 mutations, including missense/nonsense SNVs and small deletions, are more common in other populations (https://ghr.nlm.nih.gov/gene/OCA2).

Prevalence of OCA2 is approximately $1: 38,000$ to $1: 40,000$ in most populations throughout the world. The exception to this is the African population, in which the prevalence is estimated at $1: 1,500$ to $1: 3,900[10,11]$. Here we report a new type of OCA in two unrelated Korean families with the same OCA2 mutation.

\section{Case}

Participants were sourced from two, unrelated Korean families comprising four generations. This study was approved by the Institutional Review Board of the Kyungpook National University Hospital (IRB No: KNUH 2016-06-011). Written informed consents were obtained from the patient's parents for publication of this case report and accompanying images.

A 4-year-old Korean male patient came to seek medical attention for genetic diagnosis of albinism. The patient had ivory skin, medium blond hair, and light brown eyes (Fig. 1A). The father of the patient was also diagnosed with OCA when he was young (Fig. 1B). On ophthalmologic examination, best-corrected visual acuity (BCVA) was 20/50 for both eyes. He presented with esotropia and reduced stereoscopic vision. The iris was hypopigmented, but iris translucency was not evident on slit lamp examination. Fundus examination showed hypopigmented fundi (Fig. 1C). The patient was born with pale white skin and blond hair, both of which had acquired increased pigmentation with age. The color of his eyes, skin and hair gradually became darker and attained normal pigmentation in his late twenties. At the time of the visit, the father showed normal black hair and tan skin (Fig. 1A). The albinism showed an autosomal dominant inheritance pattern through the history of the family: the father, one of his aunts, grandfather, and great-grand mother (Fig. 1D).
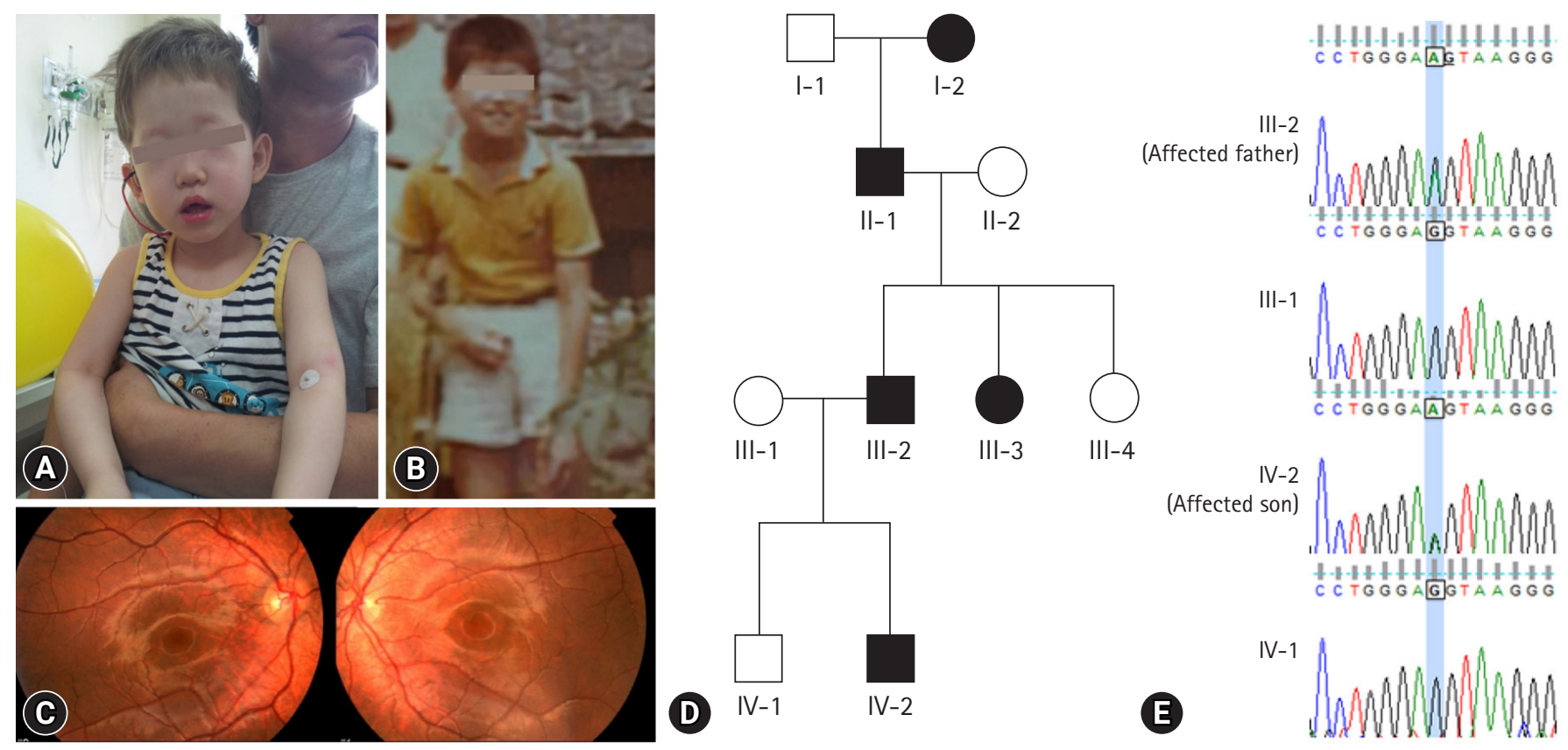

Fig. 1. (A) General appearance of the affected son and the affected father of the first family at the time of visit. (B) The father of the patient also had oculocutaneous albinism when he was young. (C) Hypopigmented fundi of the affected son. (D) The pedigree shows autosomal dominant inheritance. (E) Chromatograms of family members showing the same OCA2 mutation in the affected individuals. 

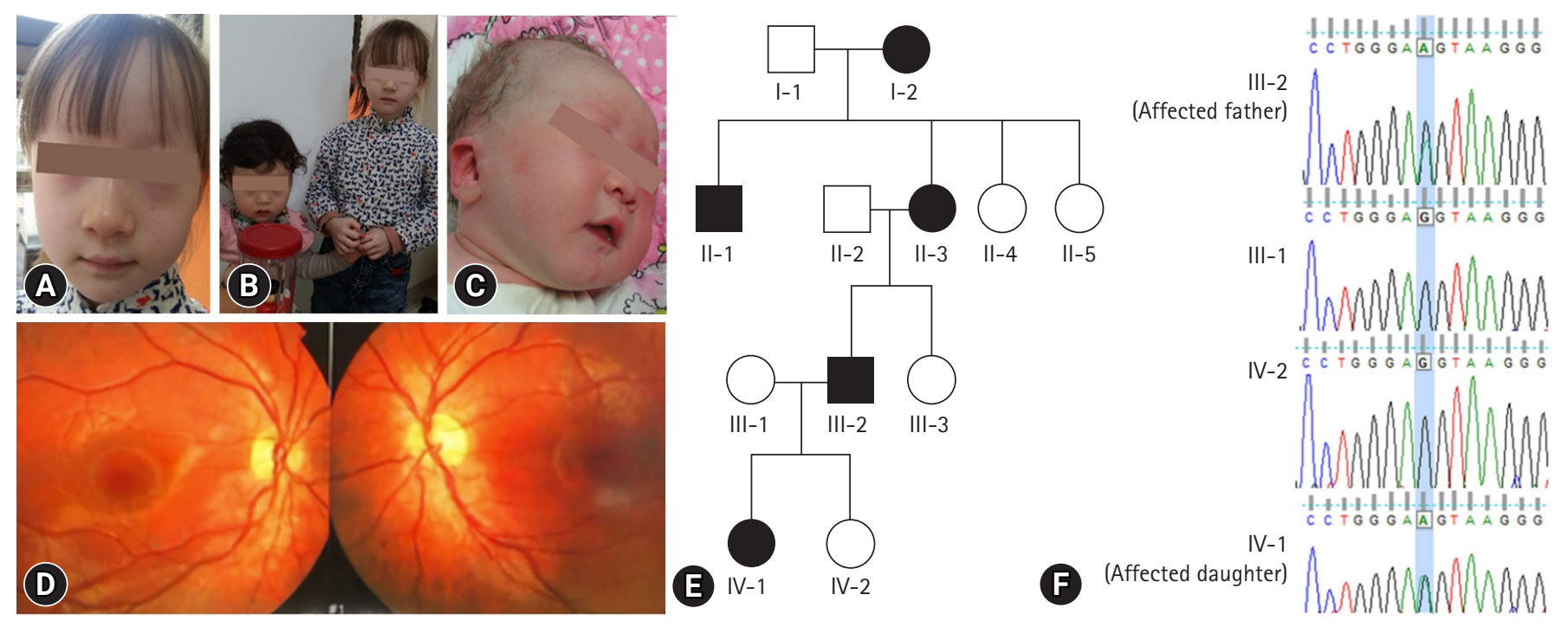

Fig. 2. (A) General appearance of the affected daughter at the time of visit. (B) General appearance of the affected daughter and her unaffected sister. (C) The affected daughter had pale white skin and blond hair when she was just born. (D) Hypopigmented fundi of the affected daughter. (E) The pedigree shows autosomal dominant inheritance. (F) Chromatograms of family members showing the same OCA2 mutation in the affected individuals.

A 6-year-old Korean female patient was referred for evaluation of hypopigmentation. She had ivory skin, dark blond hair, and light brown eyes (Fig. 2A). Her younger sister had normal pigmentation (Fig. 2B). She had pale white skin and blond hair when she was born (Fig. 2C). On ophthalmologic examination, BCVA was 20/25 for both eyes and fundus examination showed hypopigmented fundi (Fig. 2D). A gradual increase in pigmentation was observed in the color of her eyes, skin and hair compared to the time of her birth. Similar to the father of the male patient described earlier, her father had OCA when he was young, the characteristics of which became darker and attained normal pigmentation in his late twenties. The albinism showed an autosomal dominant inheritance pattern through the father, grandmother, great-granduncle, and great-grandmother (Fig. 2E). The affected individuals had similar characteristics which displayed progressive darkening and completely normal pigmentation by their late twenties.

Whole exome sequencing and in-silico analysis revealed a novel mutation, OCA2 c.2338G > A p.(G780S) (NM_000275). In-silico analysis was performed using bioinformatics software programs such as SIFT (http://sift.jcvi.org/), polyPhen-2 (http:// genetics. bwh.harvard.edu/pph2/), and phastCons score. Prediction results were deleterious (0.00) in SIFT and damaging (1.00) in polyPhen-2, indicating that the amino-acid substitutions have a high probability of pathogenicity. G780S was extremely conserved in multiple species with phastCons scores of 1.0, and has never been reported in the HGMD, HGMD Professional database, dbSNP, or the 1000 Genomes database with a minor allele frequency of 0 . Sanger sequencing of p.G780S validated the same mutation in the affected individuals which was not identified in the family members with normal phenotype (Fig. 1E, 2F).

\section{Discussion}

OCA2 encodes a pigment cell-specific, 12-transmembrane domain protein with homology to ion permeases. G780S is located in one of the extracellular topological domains of OCA2. Several studies demonstrated the function of $O C A 2$ as a positive regulator of $\mathrm{pH}$ neutralization and melanogenesis promotion $[12,13]$. TYR is the key enzyme for melanogenesis which is solely dependent on neutral $\mathrm{pH}$. Ion transport proteins such as OCA2, SLC45A2, and TPC2 function together to promote TYR function in a so called "combinational function of ion transport proteins" for the maintenance of $\mathrm{pH}[14]$. OCA2 induces large outward $\mathrm{Cl}$ - conductance which is essential for maintaining neutral $\mathrm{pH}$ for melanosomes [14]. SLC45A2 and TPC2 act as modifier genes of OCA2. Modifier genes are defined as genes that affect the phenotypic properties of other genes. Genetic modifiers can affect penetrance, dominance, expressivity, and pleiotropy. The $\mathrm{P}$ protein is not composed of dimers and seems unlikely to have a dominant-negative effect. It might represent a gain-of-function due to other historical genetic factors of melanogenesis in these families.

Known pathogenic mutations of OCA2 include V443I, P743L, and A481T. Patients with compound heterozygous mutations of V443I and P743L had severe OCA2 phenotypes [15]. OCA2 A481T has previously been reported to result in partial function of the $P$ protein [15-17]. Melanin production gradually increased in 
patients with OCA2 A481T during childhood, but no case had been reported where a patient attained normal pigmentation. The patients with OCA2 A481T had a compound heterozygous pathogenic OCA2 mutation in the other allele, and heterozygous carriers of OCA2 A481T did not show hypopigmentation [18]. Most carriers are normally asymptomatic as pathologic mutation in one copy of the OCA2 gene does not result in OCA. However, some individuals have been reported to have mild phenotypes, such as a small degree of iris transillumination, or hair and skin hypopigmentation in a heterozygous state [18]. In another study, carriers of N476D of OCA2 presented with a mild hypopigmentation phenotype, which was particularly obvious at a young age [19].

Affected individuals in this study are different from those previously reported in two aspects: an inheritance pattern and clinical presentation. All reported patients with OCA to-date have shown an autosomal recessive inheritance pattern, while our patients showed an autosomal dominant inheritance pattern. There has never been a reported case of a patient who attained normal pigmentation, while our patients displayed completely normal pigmentation in their late twenties. Individuals with $O C A 2$ mutation can acquire small amounts of pigment with age, but this does not vary substantially from adolescence to adulthood [20].

In summary, we report a novel OCA2 gene mutation found in two OCA families. We hypothesize that OCA2 G780S not only acts as a pathogenic variant of OCA, but also induces pigmentation by enhancing melanogenesis gene expression of other modifier genes, such as SLC45A2 and TPC2. These findings may provide further understanding of melanin biosynthesis and new treatments of OCA.

\section{Acknowledgments}

\section{Conflicts of interest}

No potential conflict of interest relevant to this article was reported.

\section{Author contributions}

Conceptualization: SYL, EJL, JCB, SYK, SKH; Data curation: KMJ, SYK, SKH; Formal analysis: KMJ, SKH; Validation: SYL; Methodology, Resources: JCB; Investigation: SYL, EJL; Software: SYK; Supervision: SKH; Writing-original draft: SYL, EJL, SKH; Writing-review \& editing: JCB, KMJ, SYK, SKH.

\section{ORCID}

Sang Yoon Lee, https://orcid.org/0000-0003-2595-505X

Eun Joo Lee, https://orcid.org/0000-0003-1014-5783

Jun Chul Byun, https://orcid.org/0000-0001-5600-6282

Kyung Mi Jang, https://orcid.org/0000-0002-2226-9268
Sae Yoon Kim, https://orcid.org/0000-0002-2610-0574

Su-Kyeong Hwang, https://orcid.org/0000-0001-8294-7094

\section{References}

1. Johanson HC, Chen W, Wicking C, Sturm RA. Inheritance of a novel mutated allele of the OCA2 gene associated with high incidence of oculocutaneous albinism in a Polynesian community. J Hum Genet 2010;55:103-11.

2. Prota G. The role of peroxidase in melanogenesis revisited. Pigment Cell Res 1992; Suppl 2:25-31.

3. Wang H, Wan Y, Yang Y, Li H, Mao L, Gao S, et al. Novel compound heterozygous mutations in OCA2 gene associated with non-syndromic oculocutaneous albinism in a Chinese Han patient: a case report. BMC Med Genet 2019;20:130.

4. Dessinioti C, Stratigos AJ, Rigopoulos D, Katsambas AD. A review of genetic disorders of hypopigmentation: lessons learned from the biology of melanocytes. Exp Dermatol 2009;18:7419.

5. Yang Q, Yi S, Li M, Xie B, Luo J, Wang J, et al. Genetic analyses of oculocutaneous albinism types 1 and 2 with four novel mutations. BMC Med Genet 2019;20:106.

6. Inagaki K, Suzuki T, Shimizu H, Ishii N, Umezawa Y, Tada J, et al. Oculocutaneous albinism type 4 is one of the most common types of albinism in Japan. Am J Hum Genet 2004;74:466-71.

7. Gardner JM, Nakatsu Y, Gondo Y, Lee S, Lyon MF, King RA, et al. The mouse pink-eyed dilution gene: association with human Prader-Willi and Angelman syndromes. Science 1992;257:11214.

8. Ramsay M, Colman MA, Stevens G, Zwane E, Kromberg J, Farrall $\mathrm{M}$, et al. The tyrosinase-positive oculocutaneous albinism locus maps to chromosome 15q11.2-q12. Am J Hum Genet 1992;51:879-84.

9. Rinchik EM, Bultman SJ, Horsthemke B, Lee ST, Strunk KM, Spritz RA, et al. A gene for the mouse pink-eyed dilution locus and for human type II oculocutaneous albinism. Nature 1993;361:72-6.

10. Stevens G, Ramsay M, Jenkins T. Oculocutaneous albinism (OCA2) in sub-Saharan Africa: distribution of the common 2.7-kb P gene deletion mutation. Hum Genet 1997;99:523-7.

11. Stevens G, van Beukering J, Jenkins T, Ramsay M. An intragenic deletion of the $\mathrm{P}$ gene is the common mutation causing tyrosinase-positive oculocutaneous albinism in southern African Negroids. Am J Hum Gene 1995;56:586-91.

12. Ancans J, Tobin DJ, Hoogduijn MJ, Smit NP, Wakamatsu K, Thody AJ. Melanosomal $\mathrm{pH}$ controls rate of melanogenesis, eumelanin/phaeomelanin ratio and melanosome maturation in 
melanocytes and melanoma cells. Exp Cell Res 2001;268:2635.

13. Ancans J, Hoogduijn MJ, Thody AJ. Melanosomal pH, pink locus protein and their roles in melanogenesis. J Invest Dermatol 2001;117:158-9.

14. Wiriyasermkul P, Moriyama S, Nagamori S. Membrane transport proteins in melanosomes: Regulation of ions for pigmentation. Biochim Biophys Acta Biomembr 2020;1862:183318.

15. Lee ST, Nicholls RD, Bundey S, Laxova R, Musarella M, Spritz RA. Mutations of the P gene in oculocutaneous albinism, ocular albinism, and Prader-Willi syndrome plus albinism. N Engl J Med 1994;330:529-34.

16. Saitoh S, Oiso N, Wada T, Narazaki O, Fukai K. Oculocutaneous albinism type 2 with a $\mathrm{P}$ gene missense mutation in a patient with Angelman syndrome. J Med Genet 2000;37:392-4.
17. Suzuki T, Miyamura Y, Matsunaga J, Shimizu H, Kawachi Y, Ohyama N, et al. Six novel P gene mutations and oculocutaneous albinism type 2 frequency in Japanese albino patients. J Invest Dermatol 2003;120:781-3.

18. Wei AH, Yang XM, Lian S, Li W. Genetic analyses of Chinese patients with digenic oculocutaneous albinism. Chin Med J 2013;126:226-30.

19. Wang Y, Wang Z, Chen M, Fan N, Yang J, Liu L, et al. Mutational analysis of the TYR and OCA2 genes in four chinese families with oculocutaneous albinism. PLoS One 2015;10:e0125651.

20. Lewis RA. Oculocutaneous albinism type 2. In: Adam MP, Ardinger HH, Pagon RA, Wallace SE, Bean LJH, Stephens K, et al., editors. GeneReviews ${ }^{\circledast}$. Seattle (WA): University of Washington; 1993. 